\title{
Modulation of the Turkey $\beta 1$-Adrenergic Receptor by Membrane Rafts - Insight from Molecular Dynamics
}

\author{
Afshan Ghumman and David Robinson* \\ School of Chemistry, University Of Nottingham, University Park, Nottingham, NG7 2RD, \\ United Kingdom. \\ * david.robinson@nottingham.ac.uk
}

Abstract: G-protein coupled receptors are targets for $\sim 70 \%$ of drugs. These receptors are responsible for signal transduction within a cell membrane (and further into the cell) and disruption to this signaling cascade is responsible for several non-infectious diseases. Membrane rafts exhibit significantly altered properties in comparison to fluid bilayers and as such may regulate certain GPCR's, or be responsible for errors in signaling. Using molecular dynamics, we have investigated the conformations of the turkey $\beta 1$-adrenergic receptor with and without an agonist in sphingolipid and cholesterol rich bilayers, typical of raft compositions, and compared these to cholesterol-poor bilayers. We find that the raft-like lipid bilayers are responsible for significantly altering the conformation of the receptor, even when an agonist is present. 
Microdomains within lipid membranes are known to have substantially different properties to normal fluid membranes. ${ }^{1-14}$ It has been postulated that such regions may be important in the modulation of membrane-bound proteins. ${ }^{1,9}$ Of these, the G-protein coupled receptors (GPCR) are responsible for signal transduction within a cell membrane. The GPCR's activate the Gprotein by subtle conformational changes of the seven trans-membrane (TM) alpha helices. Such changes are dependent on the membrane environment and as such the lipid composition and thus the membrane fluidity, bilayer thickness and dipole potential ${ }^{15}$ could modulate the activity of the GPCR's. If the GPCR is significantly affected by the membrane raft, then it is possible that signaling defects could occur. In this work, we consider the turkey $\beta 1$-adrenergic receptor which is usually found in non-raft membrane regions. ${ }^{16}$ We consider the effect of this receptor in the presence of the agonist epinephrine.

We have considered three different membrane environments: a pure sphingomyelin bilayer, a mixed sphingomyelin/cholesterol bilayer (referred to herein as "raft-like") and a mixed sphingomyelin/cholesterol bilayer with a phase boundary (see Methods and Supporting Information for further details). Each of these were considered in the apo and holo forms (with epinephrine). Given in Figure 1 are the helix tilt angles with respect to the membrane normal for each of the seven trans-membrane (TM) helices, along with differences from between the apo and holo structures. We first note that the X-ray crystal structure is for the receptor with a bound antagonist (cyanopindolol), and is thus found in a non-active conformation. ${ }^{16}$ The pure sphingomyelin bilayer, with and without epinephrine, are different from the X-ray structure, as one would expect for the non-antagonist bound form of the receptor. We note, however, that the phase-boundary simulation data shows close similarity to the crystal structure, especially for helices 3-5, which are known to be critical to receptor activation. ${ }^{16}$ Given these data, we expect the receptor to be activated within the noncholesterol bilayer (upon binding epinephrine), and hence we analyse the other bilayers further with this assumption. Changes in the conformation of the helices, especially helices 3 and 4, are quite substantial in the non-cholesterol bilayer (Figure 1b), while the phasechange bilayer exhibits virtually no change in conformation upon binding. The RMSF values for loop residues of the intracellular loops, IC2 and IC3, indicate some ordering when the agonist is bound within the pure sphingomyelin bilayer, with a smaller RMSF of the loop residues over the course of the simulation compared to the non-bound form (IC2: $1.27 \AA$ and $0.80 \AA$, for unbound and bound; IC3: $1.43 \AA$ and $1.24 \AA$ ). However, no change is observed for IC2 and the opposite trend seen for IC3 within the raft-like bilayer. One of the extracellular loops, EC2, exhibits opposing trends, with a small ordering on agonist activation in the pure sphingomyelin bilayer, but more significant disordering in the cholesterol-rich bilayers. 

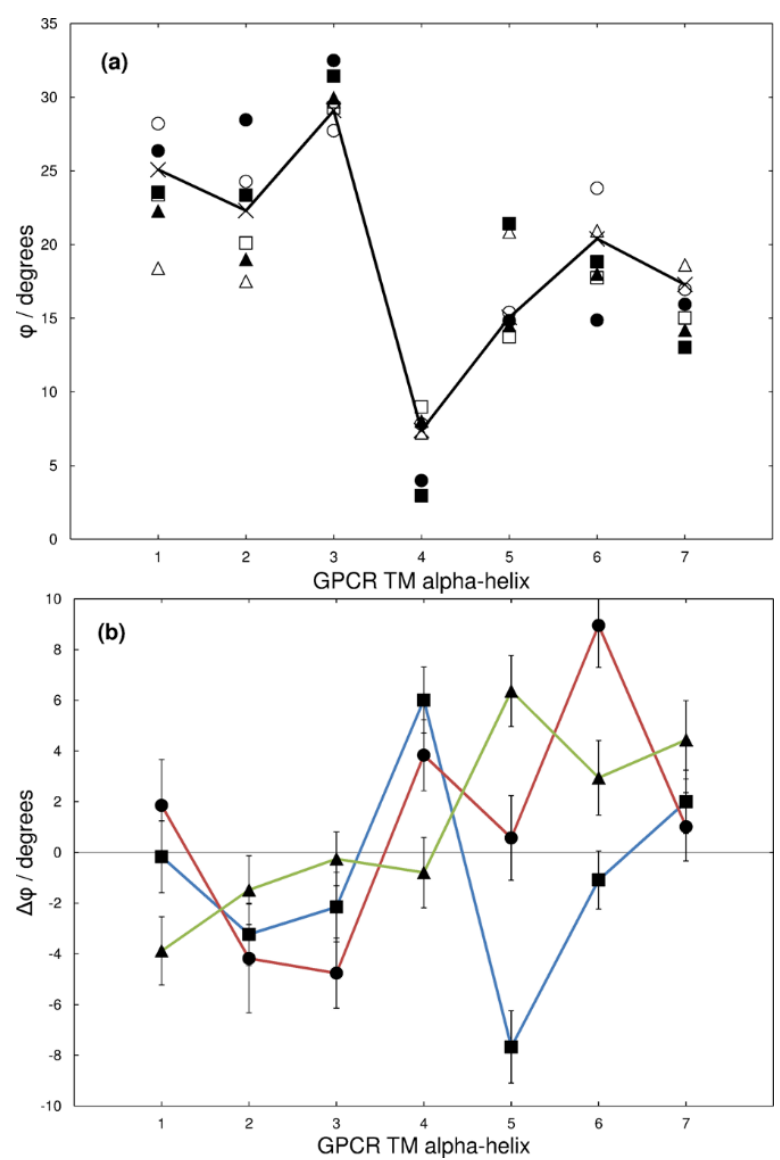

Figure 1. (a) Helix tilt angles for each of the seven TM helices with respect to the membrane normal. (b) Angle differences upon binding of the agonist. MD simulations: pure sphingomyelin simulation, filled circles; pure sphingomyelin with epinephrine, open circles; mixed sphingomyelin/cholesterol, filled squares; mixed sphingomyelin/cholesterol with epinephrine, open squares; Phase-change, filled triangles; Phasechange with epinephrine, open triangles; X-ray crystal structure, crosses with connecting lines (1a only).

The average lipid residence times, defined as the time a lipid is associated with the receptor (within $5 \AA$ ) before diffusing away, are given in Table 1. The overall average residence times are $\sim 6 \mathrm{~ns}$, with cholesterol showing a slightly longer residence time than sphingomyelin. Those lipids adjacent to the receptor have much longer residence times than the average values in Table 1, at over 20 ns (not shown in the table); these correspond to the first "solvation" layer of lipids. The second solvation layer exhibits somewhat different behavior; the average residence time for cholesterol is $\sim 0.7 \mathrm{~ns}$, while for sphingomyelin it is $0.4 \mathrm{~ns}$. 
Table 1. Ensemble averaged residence times for the lipids to associate with the receptor.

\begin{tabular}{lll}
\hline Membrane bilayer ${ }^{[a]}$ & $<$ tres $>(S S M) / n s$ & $<t_{\text {res }}>(C h o l) / n s$ \\
\hline SSM & 6.0 & - \\
SSM/Chol & 5.9 & 6.3 \\
SSM/Chol (phase change) & 6.4 & 6.1 \\
\hline
\end{tabular}

[a] SSM - pure sphingomyelin bilayer; SSM/Chol - 30 molar \% of cholesterol; $\mathrm{SSM} / \mathrm{Chol}$ (phase change) -30 molar \% of cholesterol, with half of the simulation box depleted in cholesterol and the other half enriched.

The boundary between the liquid-ordered $\left(l_{0}\right)$ and liquid disordered $\left(I_{d}\right)$ lipids is where many membrane proteins are found. Given in Figure 2 is a snapshot from the simulation with a phase-boundary, showing the propensity of cholesterol molecules to surround the receptor from the $I_{0}$ side, with sphingomyelin dominating the lipid phase on the opposite side of the receptor. This is an interesting insight and would benefit from studies using larger membrane patches and longer timescales than the present study, with associated development of mixed-resolution MD approaches. ${ }^{17,18}$

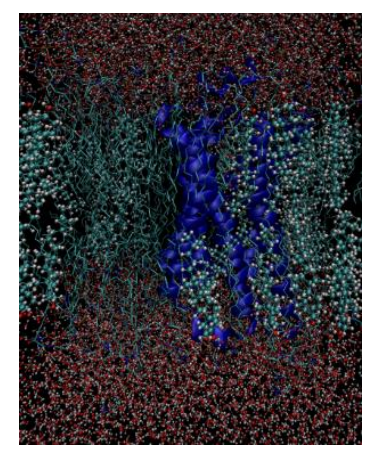

Figure 2. Snapshot of the membrane from the phase-boundary simulation. The membrane normal is the vertical axis and the " $y$ "-axis is the horizontal. The receptor is shown in cartoon representation, the sphingomyelin molecules as lines and cholesterol molecules using a ball-and-stick representation. The $l_{d}$ phase is on the left of the receptor, with the $l_{0}$ phase on the right.

Given in Figure 3 is a typical snapshot of the binding site and hydrogen bond interactions with the epinephrine molecule. Previous MD simulations ${ }^{19}$ and X-ray crystallography studies ${ }^{20}$ indicate a strong preference for the epinephrine molecule to hydrogen-bond, via the $-\mathrm{NH} 2$ group, to the two oxygen atoms of Asp121. We observe this bonding pattern only in the pure sphingomyelin simulations, with average hydrogen bond lifetimes of $\sim 60 \mathrm{ps}$. However, in the cholesterol-rich membranes, the - $\mathrm{NH} 2$ group hydrogen bonds to both Asp121 and Arg329, with the average hydrogen bond lifetime being much longer than observed in the pure sphingomyelin simulation, $\sim 400$ ps. The average lifetime of the hydrogen-bond increases further in the phase-boundary simulation, to $>600 \mathrm{ps}$. In all of the simulations, hydrogen- 
bonding of the epinephrine molecule to water molecules is also observed, as expected given the solvent-exposed nature of the active site of the receptor. The apo form of the receptor in the cholesterol-rich bilayers shows different conformations of the TM helices than is the case for the pure sphingomyelin bilayer, with a calculated molecular volume of the active site somewhat smaller than for the sphingomyelin bilayer. This feature is also observed in the holo form of the receptor; subsequently, the epinephrine molecule has to alter its orientation in order to avoid being ejected from the active site. While this results in much stronger binding to residues within the active site, these are not the residues known to activate the receptor. ${ }^{19,20}$

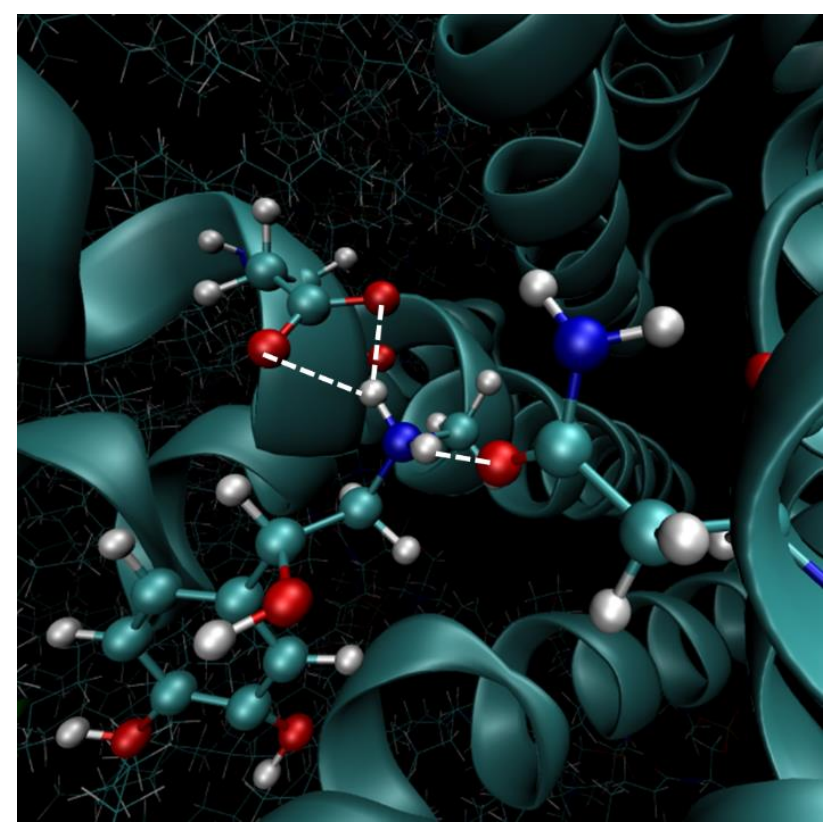

Figure 3. Active site hydrogen bonding to epinephrine (taken from the phase-boundary simulation).

Using molecular dynamics simulations, we have shown that the apo form of the turkey $\beta 1$-adrenergic GPCR exhibits significant structural differences in non-raft and raft-like membrane compositions, and this is also true for the agonist-bound form of the receptor. Importantly, the structure in the raft-like membrane for the apo form is consistent with the antagonist-bound form of the receptor. Within a system with a "phase boundary", similar conformational changes are observed. Interestingly, the receptor preferentially binds to cholesterol molecules in this boundary region. It should be noted that the effect of sodium ions has not been included in this work. Other studies have shown that sodium ions play an important role in the stabilisation of apo- $\beta 1$ adrenoreceptors. ${ }^{21}$ These results shed some light on the modulation of the receptor by the membrane raft, hinting at possible signalling defect mechanisms; further computational work using larger membrane patches and a wider range of receptors is needed to enhance our understanding, complementary to experimental studies. 


\section{Methods}

Molecular dynamics simulations were performed on six membrane systems: a pure sphingomyelin bilayer with the turkey $\beta 1$ adrenergic GPCR (PDB code: 2VT4);16 pure sphingomyelin with receptor and epinephrine; ${ }^{19}$ mixed bilayer of sphingomyelin (70 molar \%) and cholesterol (30 molar \%) with receptor; mixed bilayer of sphingomyelin (70 molar \%) and cholesterol (30 molar \%) with receptor and epinephrine. The same mixed bilayer system was used for a further simulation, emulating a phasechange by having half of the simulation box cholesterol-enriched and the other half cholesterol depleted (see Supporting Information for full details of the procedure used). The epinephrine molecule was inserted into the GPCR according to the interactions seen by Wacker et al. ${ }^{20}$ Equilibration was performed for $20 \mathrm{~ns}$, with production dynamics of $100 \mathrm{~ns}^{22}$ for each system. The CHARMM-GUI ${ }^{23}$, $\mathrm{CHARMM}^{24}$ and $\mathrm{NAMD}^{25}$ software packages were employed. Full computational details can be found in the Supporting Information. Analysis was performed over the last 20 ns of simulation data.

\section{Acknowledgements}

We thank the University Of Nottingham for provision of time on the Minerva High Performance Computing Cluster.

Keywords: GPCR • Membrane raft • molecular dynamics • Signalling • Agonist binding

\section{References}

[1] K. Simons, D. Toomre, Nat. Rev. Mol. Cell Biol. 2000, 1, 31-39.

[2] G. J. Schütz, G. Kada, V. P. Pastushenko, H. Schindler, EMBO J. 2000, 19, 892-901.

[3] A. Pralle, P. Keller, E. L. Florin, K. Simons, J. K. H. Hörber, J. Cell Biol. 2000, 148, 997-1007.

[4] E. Ikonen, Curr. Opin. Cell Biol. 2001, 13, 470-477.

[5] K. Gaus, E. Gratton, E. P. W. Kable, A. S. Jones, I. Gelissen, L. Kritharides, W. Jessup, Proc. Natl. Acad. Sci. USA 2003, 100, 15554-15559.

[6] R. Ishitsuka, S. B. Sato, T. Kobayashi, J. Biochem. 2005, 137, 249-254.

[7] L. J. Pike, J. Lipid Res. 2008, 50, S323-S328.

[8] J. Zidar, F. Merzel, M. Hodoscek, K. Rebolj, K. Sepcic, P. Macek, D. Janezic, J. Phys. Chem. B 2009, 113, $15795-15802$.

[9] A. A. Gurtovenko, J. Anwar, I. Vattulainen, Chem. Rev. 2010, 110, 6077-6103.

[10] M. Mihailescu, R. G. Vaswani, E. Jardón-Valadez, F. Castro-Román, J. A. Freites, D. L. Worcester, A. R. Chamberlin, D. J. Tobias, S. H. White, Biophys. J. 2011, 100, 1455-1462.

[11] J. W. O'Connor, J. B. Klauda, J. Phys. Chem. B 2011, 115, 6455-6464.

[12] J. F. Frisz, K. Y. Lou, H. A. Klitzing, W. P. Hanafin, V. Lizunov, R. L. Wilson, K. J. Carpenter, R. Kim, I. D. Hutcheon, J. Zimmerberg, P. K. Weber, M. L. Kraft, Proc. Natl. Acad. Sci. USA 2013, 110, E613-E622.

[13] M. Carquin, L. Conrard, H. Pollet, P. Van Der Smissen, A. Cominelli, M. Veiga-da-Cunha, P. J. Courtoy, D. Tyteca, Cell. Mol. Life Sci. 2015, 72, 4633-4651.

[14] Y. -W. Jiang, H. -Y. Guo, Z. Chen, Z. -W. Yu, Z. Wang, F. -G. Wu, Langmuir 2016, 32, 67396745.

[15] D. Robinson, N. A. Besley, P. O'Shea, J. D. Hirst, J. Comput. Chem. 2011, 32, 2613-2618.

[16] A. Warne, M. J. Serrano-Vega, J. G. Baker, R. Moukhametzianov, P. C. Edwards, R. Henderson, A. G. W. Leslie, C. G. Tate, G. F. X. Schertler, Nature 2008, 454, 486-491.

[17] M. Orsi, J. W. Essex, PLoS ONE 2011, 6, e28637.

[18] M. Orsi, W. Ding, M. Palaiokostas, J. Chem. Theory Comput. 2014, 10, 4684-4693.

[19] P. Spijker, N. Vaidehi, P. L. Freddolino, P. A. Hilbers, W. A. Goddard III, Proc. Natl. Acad. Sci. USA 2006, 103, 4882-4887.

[20] D. Wacker, G. Fenalti, M. A. Brown, V. Katritch, R. Abagyan, V. Cherezov, R. C. Stevens, J. Am. Chem. Soc. 2010, 132, 11443-11445.

[21] J. L. Miller-Gallacher, R. Nehmé, T. Warner, P. C. Edwards, G. F. X. Schertler, A. G. W. Leslie, C. G. Tate, PLoS One 2014, 9, e92727.

[22] D. Robinson, J. Chem. Theory Comput. 2013, 9, 2498-2503.

[23] S. Jo, T. Kim, V. G. Iyer, W. Im, J. Comput. Chem. 2008, 29, 1859-1865.

[24] B. R. Brooks, C. L. Brooks III, A. D. Mackerell, et al., J. Comput. Chem. 2009, 30, 1545-1615.

[25] J. C. Phillips, R. Braun, et al., J. Comput. Chem. 2005, 26, 1781-1802. 